\title{
Die wundersame Flora rund um die kambodschanische Tempelstadt Angkor
}

\author{
Rolf Goetz
}

\author{
„Der König braucht das Volk, der Mönch braucht die Regeln, der Fisch braucht das Wasser“. \\ Sprichwort der Khmer
}

\begin{abstract}
The temple city of Angkor is often called the eighth wonder of the world. The UNESCO World Heritage is located about $300 \mathrm{~km}$ northwest of the Cambodian capital Phnom Penh on the outskirts of the provincial capital Siem Reap. The architecture, dating back to the 12th century, with outstanding testimonies of Hindu Buddhist culture makes Angkor a much visited sight. Among the approximately 1000 (!) temple complexes are especially Angkor Wat, Angkor Thom and Ta Prohm to emphasize. The focus of this article is on the tropical "temple flora" around the sprawling archeological district.
\end{abstract}

\section{Zusammenfassung}

Die Tempelstadt Angkor wird vielfach achtes Weltwunder genannt. Das UNESCO-Welterbe liegt gut $300 \mathrm{~km}$ nordwestlich der kambodschanischen Hauptstadt Phnom Penh am Rand der Provinzmetropole Siem Reap. Die bis ins 12. Jahrhundert zurückreichende Baukunst mit herausragenden Zeugnissen hinduistisch-buddhistischer Kultur macht Angkor zu einer viel besuchten Sehenswürdigkeit. Unter den rund 1000 (!) Tempelanlagen sind vor allem Angkor Wat, Angkor Thom und Ta Prohm hervorzuheben. Der Fokus dieses Artikels richtet sich auf die tropische „Tempelflora“ rund um den weitläufigen Aachäologischen Bezirk.

\section{Einführung}

Wie der ganze Tropengürtel Südostasiens war Kambodscha einmal von dichten Regenwäldern überzogen. Mehr als die Hälfte des Waldes fiel dem

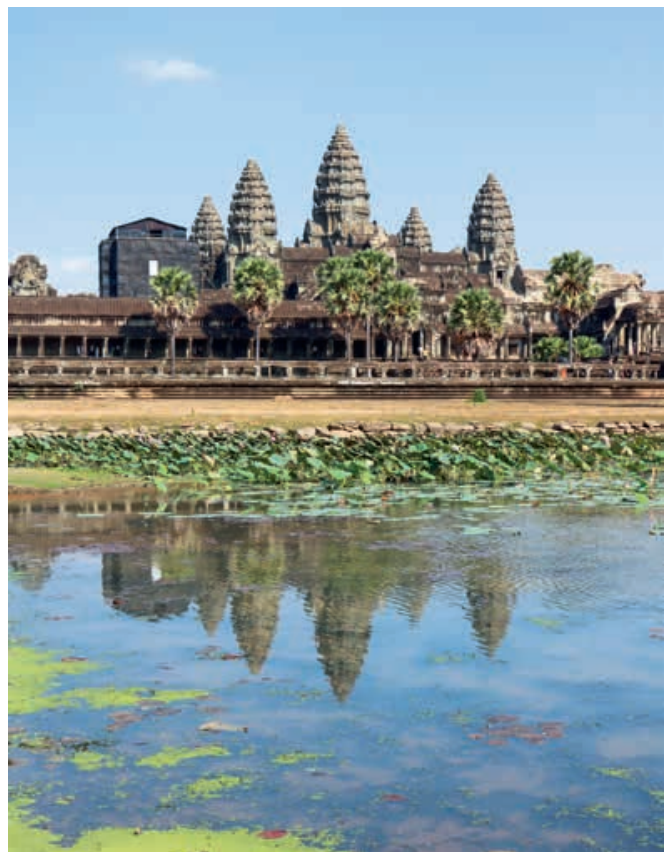

Abb. 1: Angkor Wat mit Lotosteich und Palmyrapalmen. (Foto: R. Goetz) hemmungslosen Raubbau des Menschen zum Opfer. Die etwa $400 \mathrm{~km}^{2}$ große Region um die Tempelstadt Angkor kam dabei noch relativ glimpflich weg. Vom 9. bis zum 15. Jahrhundert war Angkor das politische und religiöse Zentrum des Königreichs der Khmer, von dem weite Teile Südostasiens kontrolliert wurden. Zur Blütezeit lebten in und um die Tempelstadt etwa eine Million Menschen, Angkor galt damals als größte Stadt der Welt. Nach dem Untergang des Khmer-Reiches, die genauen Hintergründe für den Zerfall sind unklar, versanken die Tempel in einen jahrhundertelangen Dornröschenschlaf. In Europa wurde man erst wieder durch den Besuch des französischen Forschungsreisenden Henri Mounot (1826- 1861) darauf aufmerksam. Der Franzose bereiste im Januar 1860 Angkor und fand damals eine vom Urwald fast völlig vereinnahmte Ruinenstadt vor. Restaurierungsarbeiten begannen 1908 unter der Leitung eines Teams der École Française d'Extrême-Orient, die ersten Touristen kamen bereits wenige Jahre später.

\section{Touristische Bedeutung}

Durch den blutigen Bürgerkrieg in den 1980er-Jahren und die Schreckensherrschaft der Roten Khmer („Killing Fields“) war Kambodscha über Jahre von der Außenwelt abgeschlossen. Seit der politischen 


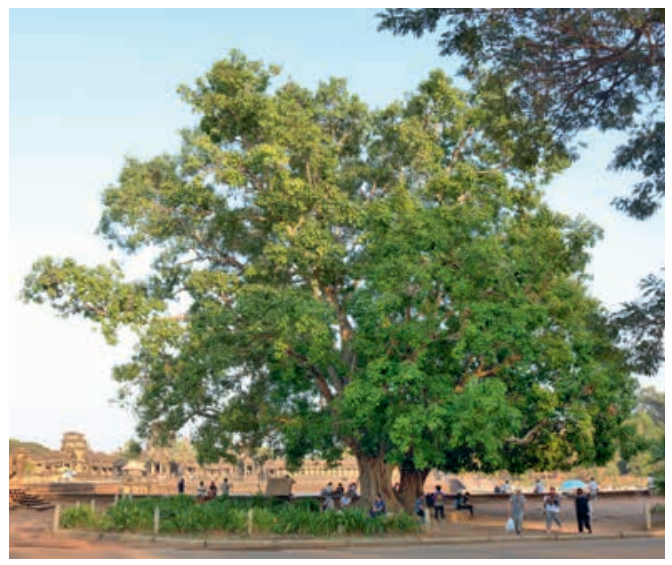

Abb. 2: Die Pappelfeige (Ficus religiosa) bietet sich für eine kleine Pause an, im Hintergrund die Silhouette von Angkor Wat. (Foto: R. Goetz)

Neuausrichtung 1992 hat sich das Land unter relativ stabilen Machtverhältnissen sichtlich erholt, es gehört jedoch immer noch zu den ärmsten in Asien. Eine wirtschaftliche Stütze ist der rasant gewachsene Tourismus. Mit etwa vier Millionen Besuchern pro Jahr avancierte die Tempelstadt Angkor zu einem Hotspot des Welttourismus (MeYers \& Markland 2018). In der nur wenige Kilometer entfernten ehemaligen französischen Kolonialstadt Siem Reap (ca. 150000 Einw.) findet das internationale Publikum - neben Chinesen und Koreanern auch viele US-Amerikaner, Franzosen und Deutsche - eine nahezu perfekt ausgebaute Infrastruktur. Außer einem internationalen Flughafen

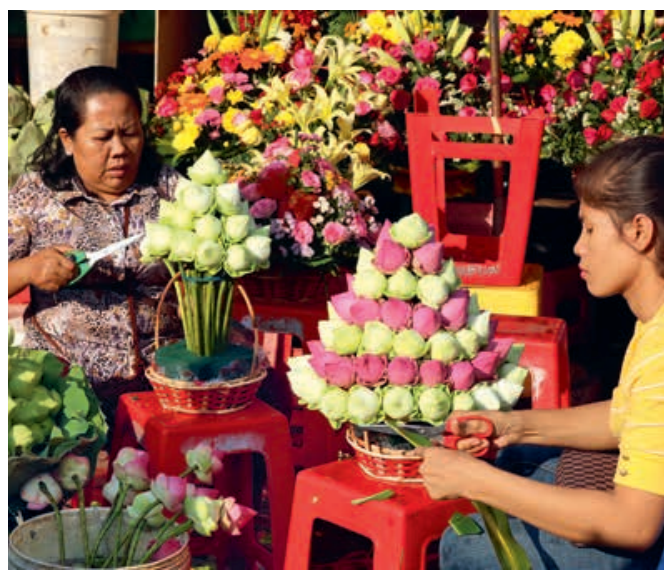

Abb. 4: Auf lokalen Blumenmärkten werden aus Lotosblüten kunstvolle Tempelgaben hergestellt. (Foto: R. Goetz)

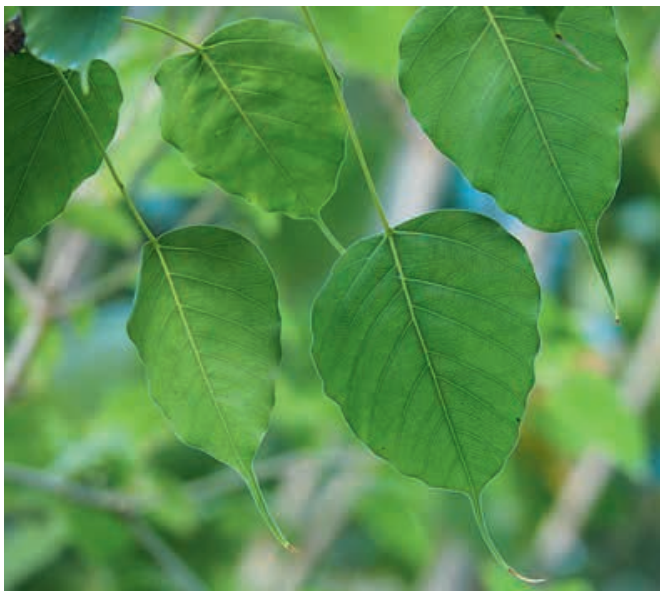

Abb. 3: Blätter von Ficus religiosa mit charakteristischer Träufelspitze. (Foto: R. Goetz)

und Unterkünften in allen Preisklassen gehört dazu auch eine genussreiche Gastronomieszene und eine lebhafte Partymeile, in der bis weit nach Mitternacht gefeiert wird. Viele Touristen besuchen Angkor im Rahmen einer Rundreise durch Südostasien; der Flughafen Siem Reap wird u. a. von Singapur, Bangkok und Ho-Chi-Minh-Stadt (Saigon) angeflogen. Staatsbürger der EU benötigen zur Einreise ein Visum, das an der Grenze ausgestellt wird (derzeit 34 US-Dollar). Ein Tagesticket für den Besuch des archäologischen Bezirks von Angkor kostet 37 US-Dollar, für eine umfassende Erkundung des weitläufigen Areals empfiehlt sich ein Drei-TagePass (62 US-Dollar).

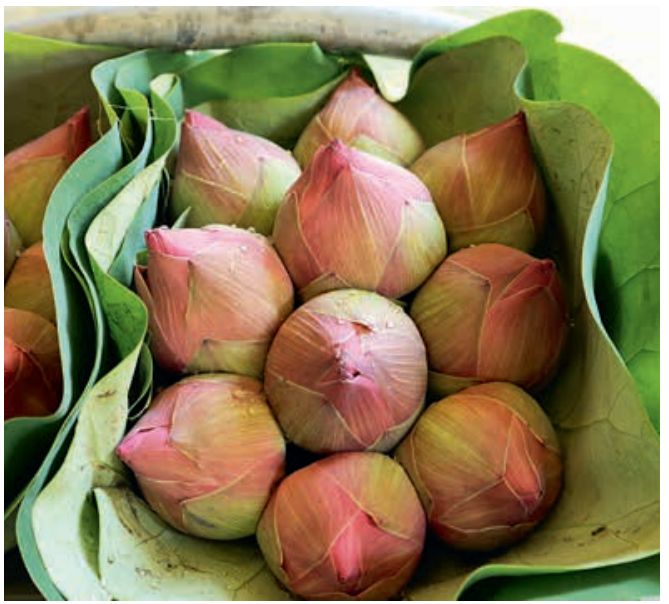

Abb. 5: Blütenknospen der Lotosblume (Nelumbo nucifera). (Foto: R. Goetz) 


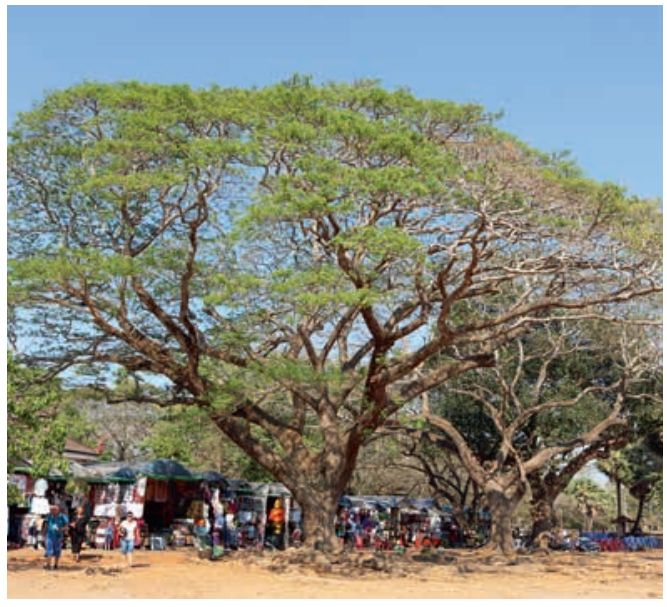

Abb. 6: Die ausladende Krone des Regenbaums (Samanea saman) spendet einer ganzen Ladenzeile Schatten. (Foto: R. Goetz)

\section{Am Haupttempel Angkor Wat: Buddhabaum und Lotosteich}

Unter welchem Baum Buddha (Siddharta Gautama, 563-483 v. Chr.) vor zweieinhalb Jahrtausenden Erleuchtung erlangte, ist nicht exakt überliefert. Vermutlich meditierte der Religionsstifter unter der ausladenden Krone einer Pappel-Feige (Ficus religiosa). Das auf dem ganzen indischen Subkontinent und in Südostasien verbreitete Maulbeergewächs wird vielfach Baum der Weisheit, Buddhabaum oder Bodhibaum (nach dem Sanskrit-Wort für Erleuchtung) genannt. In buddhistisch geprägten Ländern finden sich Pappel-Feigen meist in unmittelbarer Nähe von Tempeln und spenden mit ihren dicht belaubten Kronen Pilgern und Besuchern gleichermaßen willkommenen Schatten. Der Baum erreicht eine Wuchshöhe von $25 \mathrm{~m}$ und mehr. Selbst für den botanischen Laien ist er leicht an den herzförmigen Blättern zu bestimmen, die in 3-5 cm langen Träufelspitzen auslaufen. Sie leiten das Wasser nach einem Monsunregen schnell und verhindern so, dass sich in dem feuchten Milieu Moose oder Flechten ansiedeln. Ein stattliches Individuum von F. religiosa steht am Westeingang des Tempelbezirks von Angkor Wat, dem Haupttempel des archäologischen Bezirks von Angkor. Hier trifft man zu jeder Tageszeit Besucher aus aller Welt, die sich auf der Steinbank rund um den mächtigen Stamm eine kleine Pause gönnen.

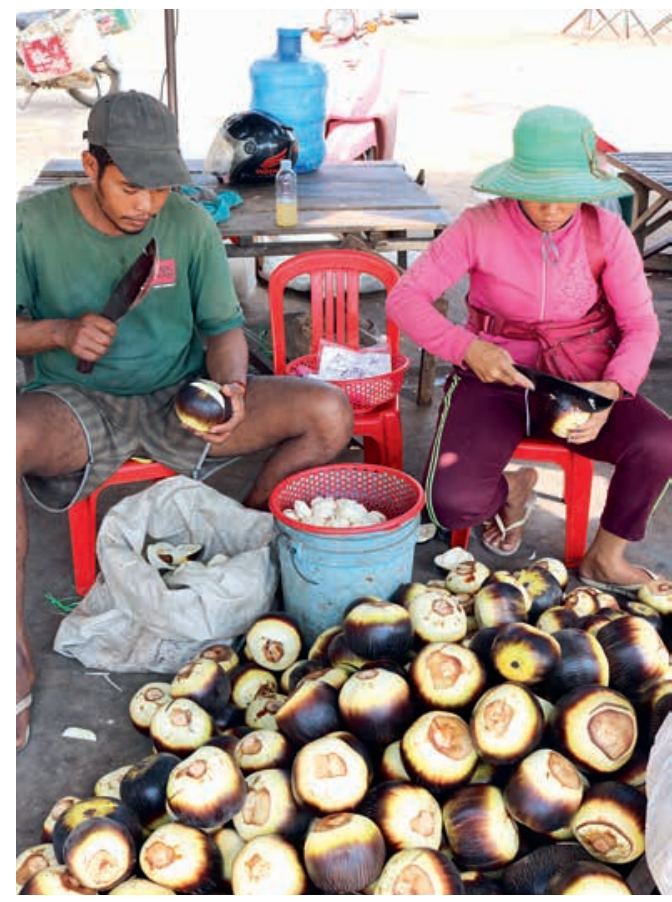

Abb. 7: Das Herauslösen der Kerne aus den Früchten der Palmyrapalme wird seit alters her in Handarbeit gemacht. (Foto: R. Goetz)

Ein paar Schritte vom Ficus entfernt führt ein Damm über einen Wassergraben ins Zentrum der Tempelanlage. Vor dieser liegt ein Tümpel, in dem sich fotogen die bis zu $55 \mathrm{~m}$ hohen Tempeltürme spiegeln. Ein kleiner Bestand an Lotosblumen (Nelumbo nucifera) gibt dem seichten Gewässer ein belebendes Element. Die Lotosblume wird in der religiösen Kultur Asiens wie kaum eine andere Pflanze verehrt. Sie steht sinnbildlich für Reinheit und Vollkommenheit, was sich u. a. ganz pragmatisch durch die von einer Wachsschicht überzogenen Blätter erklärt. Diese lassen Schmutz und Wasser abperlen, sodass die Wasserpflanze immer sauber aussieht. Im Buddhismus gehört die Lotosblume zu den acht Glückssymbolen. Buddha wird vielfach sitzend (im Lotossitz) auf einer Lotosblüte dargestellt. Auf vielen Marktständen in Kambodscha bietet man Lotosblumen für wenig Geld an. Die Verkäuferinnen verstehen es, mit wenigen Handgriffen aus den noch nicht geöffneten Blütenknospen kleine Kunstwerke herzustellen, die dann als Opfergabe den Ahnen und Gottheiten dargeboten werden. Der Lotos ist darüber 


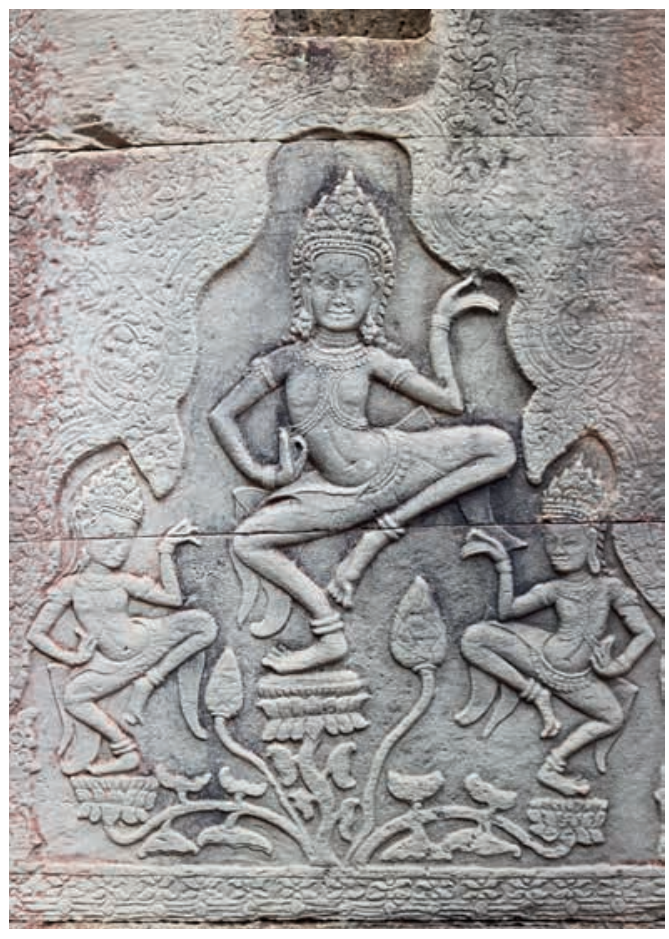

Abb. 8: Ein Flachrelief in Angkor Thom zeigt anmutig tanzende Apsaras auf einer Lotosblüte. (Foto: R. Goetz)

hinaus auch Nahrungspflanze. Die haselnussgroßen Samen (sogenannte Lotosnüsse) verzehrt man als Snack, das von kleinen Röhren durchzogene stärkereiche Rhizom (Lotoswurzel) dient als Suppeneinlage oder wird zu Mehl verarbeitet. Auch für die schildförmigen runden Blätter mit einem Durchmesser von bis zu $60 \mathrm{~cm}$ findet sich eine Verwendung, sie dienen beispielsweise als Verpackungsmaterial für Lebensmittel.

\section{Regenbaum und Palmyrapalme}

An der nördlichen Begrenzungsmauer von Angkor Wat steht eine Reihe von prächtigen Regenbäumen (Samanea saman), unter deren ausladenden Kronen sich eine Souvenirzone mit Dutzenden kleiner Verkaufsstände angesiedelt hat. Die Schirmkrone macht das ursprünglich in Mittelund Südamerika beheimatete Mimosengewächs zu einem der auffälligsten Tropenbäume, einzelne Individuen bringen es auf eine über $60 \mathrm{~m}$ breite Krone. Der Name des Schattenbaums wird darauf zurückgeführt, dass es unter ihm selbst bei nur leichten Niederschlägen immer feucht ist. Grund

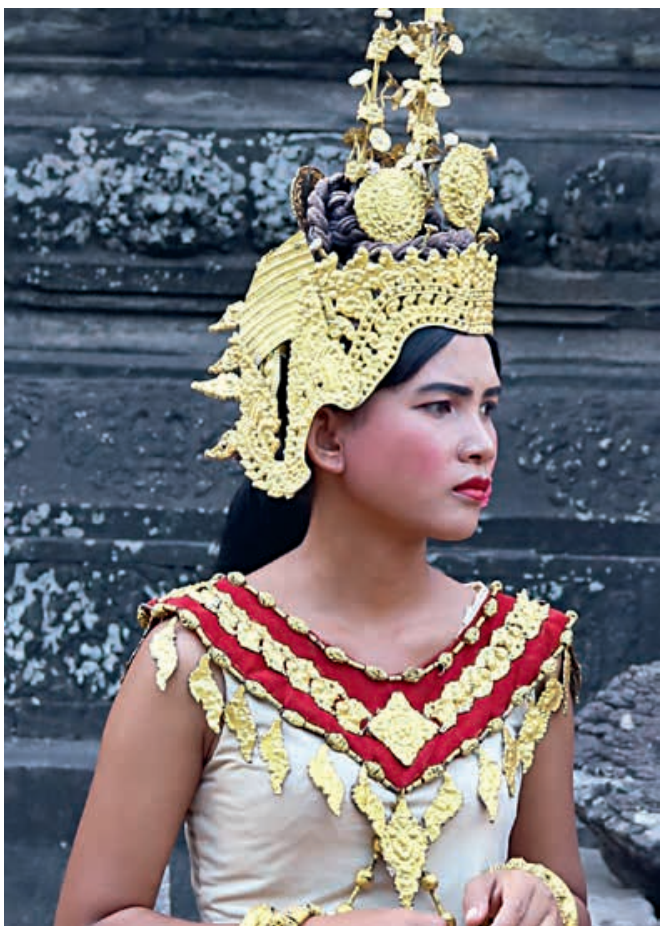

Abb. 9: Am traditionellen Vorbild orientierte moderne Apsara-Tänzerin in Angkor Wat. (Foto: R. Goetz)

dafür sind die gefiederten Laubblätter, die sich bei Regenwetter, aber auch nachts zusammenfalten und die Krone so durchlässig für Regentropfen machen (Sснӥтт 2014). Die Blätter nehmen ihre Schlafstellung kurz vor Sonnenuntergang ein und behalten diese bis kurz vor Sonnenaufgang bei. Zur Hauptblütezeit (Mai/Juni) geben die köpfchenförmigen Blütenstände mit ihren pinkfarbenen Kronröhren ein pittoreskes Bild ab.

Das kunstgeschichtliche Highlight im Tempel Angkor Wat sind detailreiche Steinbildhauerarbeiten. Besonderes Interesse genießen rund 1850 tanzende Apsaras (himmlische Wesen) auf wie in einer Galerie aufgereihten fast $100 \mathrm{~m}$ langen Flachreliefs. Etliche der Sandsteinreliefs sind vom Zahn der Zeit mehr oder weniger stark verwittert. Um die Konservierung bemüht sich seit 1995 das German Apsara Conservation Projekt (GACP) der kulturwissenschaftlichen Fakultät der Technischen Hochschule Köln. Das Team unterhält in der nordwestlichen Ecke des Tempelbezirks ein kleines Infobüro. 


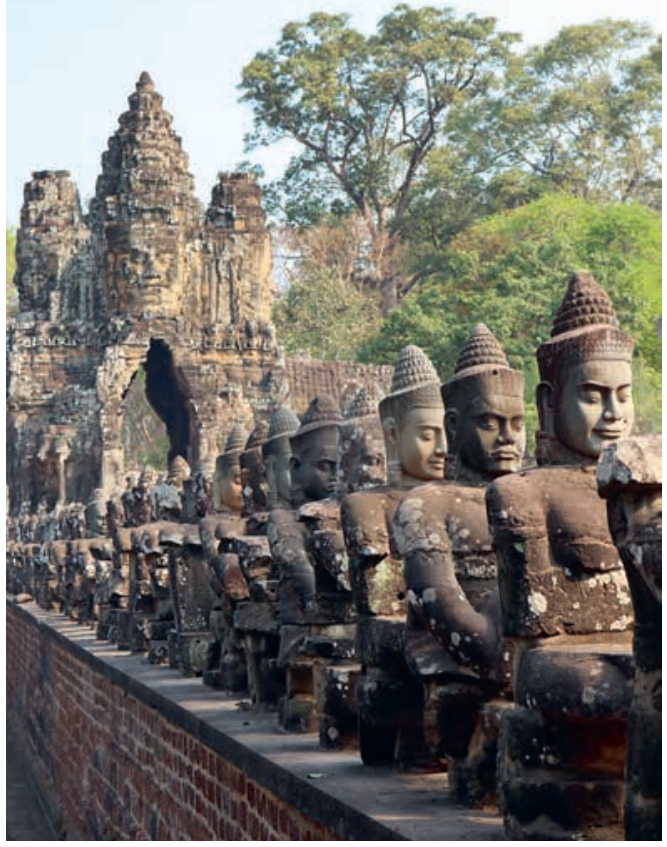

Abb. 10: Eine Balustrade aus Buddhafiguren säumt vor dem Südtor von Angkor Thom den Zugang in den Tempelbezirk. (Foto: R. Goetz)

In Angkor Wat sollte man es sich nicht nehmen lassen auf einer allerdings ziemlich steilen Treppe auf die oberste Ebene des Bauwerks zu steigen. Vor allem in der Hauptsaison im Winter herrscht ein großer Andrang, der Zugang wurde mittlerweile reglementiert und es ist mit Wartezeiten von mitunter mehr als einer halben Stunde zu rechnen. Oben angekommen wird man für die Mühe mit einem weiten Ausblick über den Dschungel belohnt. In dem grünen Meer setzen hoch aufragende Palmyrapalmen (Borassus flabellifer) Akzente. Die an die $30 \mathrm{~m}$ hohe und oft alleinstehende Fächerpalme ist in Kambodscha landschaftsprägend; sie ist der Nationalbaum des Landes und wächst vielerorts wild. Wie auch die Kokospalme wird die Palmyrapalme Baum des Lebens genannt, da alle Teile genutzt werden können: der Stamm gibt ein begehrtes Bauholz ab, die Wedel dienen als Deckmaterial für Häuser, der zuckerreiche Saft wird zu Palmzucker oder Palmwein verarbeitet und die Steinfrüchte (sogenannte Nüsse) enthalten essbare Kerne. Schon vor 1500 Jahren benutzte man die Palmblätter als Beschreibstoff. Dazu wurden die

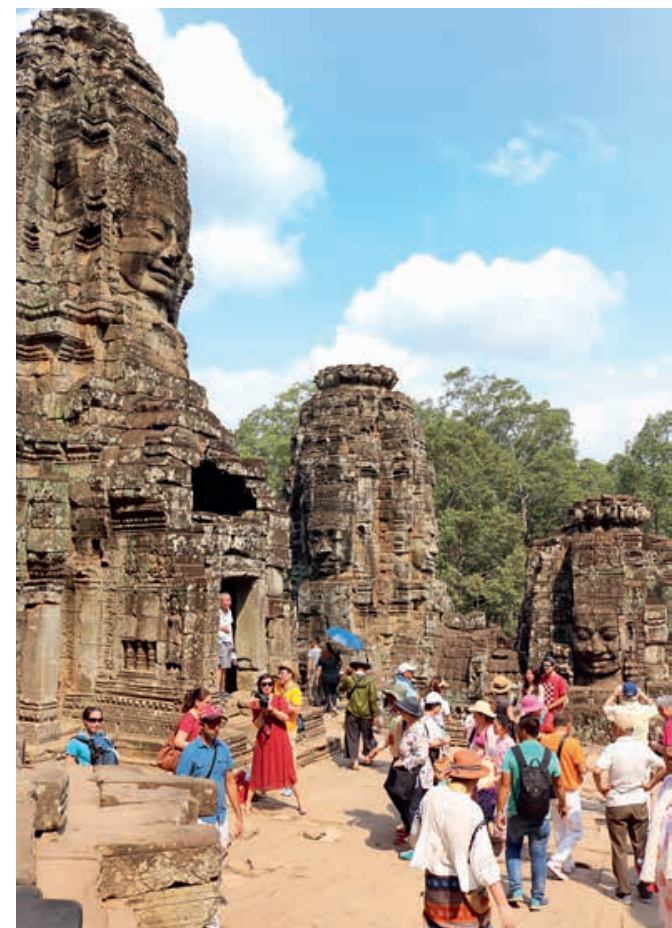

Abb. 11: Spektakuläre Baukunst: die berühmten Gesichtertürme von Angkor Thom. (Foto: R. Goetz)

Blätter in rechteckige Streifen geschnitten, die man dann zu einem größeren Blatt zusammennähte (CHAN 2003).

\section{Der Tempelbezirk von Angkor Thom}

Etwa ein Kilometer nördlich von Angkor Wat liegt mit Angkor Thom die größte Anlage des archäologischen Parks. Von dem buddhistischen Staatstempel wurde ab dem 12. Jahrhundert das Reich der Khmer verwaltet. Seine berühmten „Gesichtertürme“ machen ihn zu einem der imposantesten Sakralbauten Südostasiens. Die Spitzen der „lächelnden“ Türme zieren von aus Sandstein geschlagene Lotosblüten. Wie auch in Angkor Wat kann man sich in Angkor Thom stundenlang in die Betrachtung der auf den Umfassungsmauern angebrachten Flachreliefs vertiefen. Sie zeigen u. a. militärische Aufmärsche und Schlachtszenen, den dekorativen Hintergrund dazu stellt fein herausgearbeitetes Laub der Birkenfeige (Ficus benjamina). Etliche betagte „Benjamini“ stehen nur wenige Schritte neben der Tempelanlage, auch finden sich dort Schleicheria oleosa, ein 


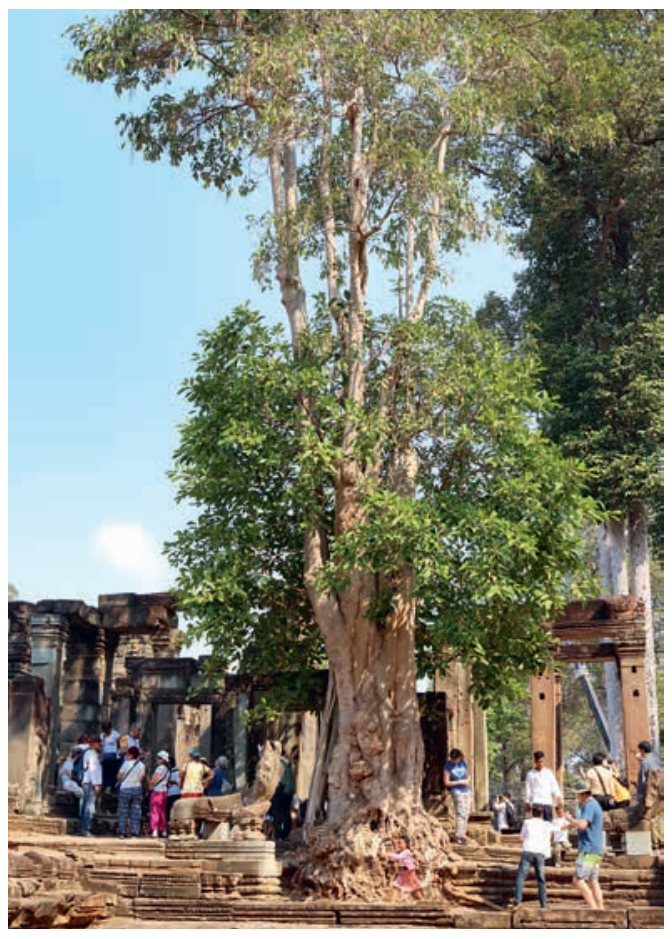

Abb. 12: Eine Birkenfeige (Ficus benjamina) vor dem Tempelbezirk Angkor Thom. (Foto: R. Goetz)

Seifenbaumgewächs, und prächtige Exemplare von hochstämmigen Lagerströmien (Lagerstroemia calyculata). In der Khmer-Sprache werden diese stolzen Bäume Sralao genannt, der deutsche Name Kräuselmyrthe (engl. crepe myrtle) leitet sich von der auffällig gesprenkelten Borke ab. Mit einem bis kurz unter die Krone astfreien Stamm macht Dipterocarpus alatus aus der Familie der Flügelfruchtgewächse auf sich aufmerksam; in der Roten Liste der IUCN wird der mehr als $50 \mathrm{~m}$ hohe Baumriese allerdings als stark gefährdet eingestuft. Nicht viel besser steht es um den ebenfalls im Tempelbezirk Angkor Thom beheimateten Hülsenfrüchtler Afzelia xylocarpa.

\section{Spungbäume und Würgerfeige im Tempel Ta Prohm}

Aus botanischer Sicht ist der Tempel Ta Prohm knapp $2 \mathrm{~km}$ östlich von Angkor Thom die Hauptattraktion. Schon auf dem Zugang durch eine Allee aus Urwaldbäumen lohnt es innezuhalten um das eine oder andere Gehölz näher zu betrachten, darunter Aglaia spectabilis und Irvingia malayana. Der

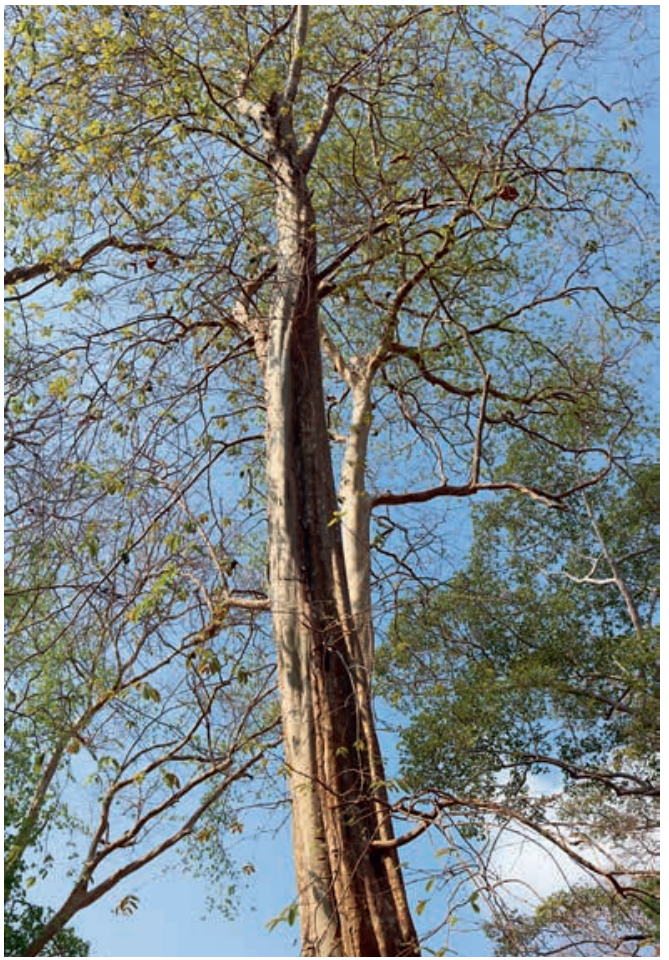

Abb. 13: Lagerstroemia calyculata. (Foto: R. Goetz)

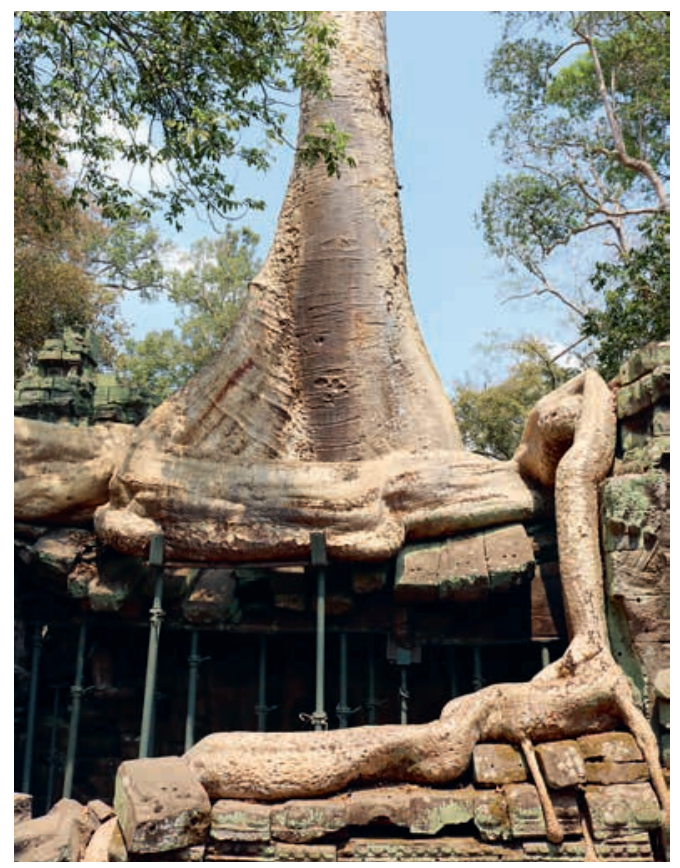

Abb. 14: Spungbaum (Tetrameles nudiflora) in Ta Prohm wie lange mag das Stützgerüst wohl noch dem tonnenschweren Gewicht standhalten? (Foto: R. Goetz) 


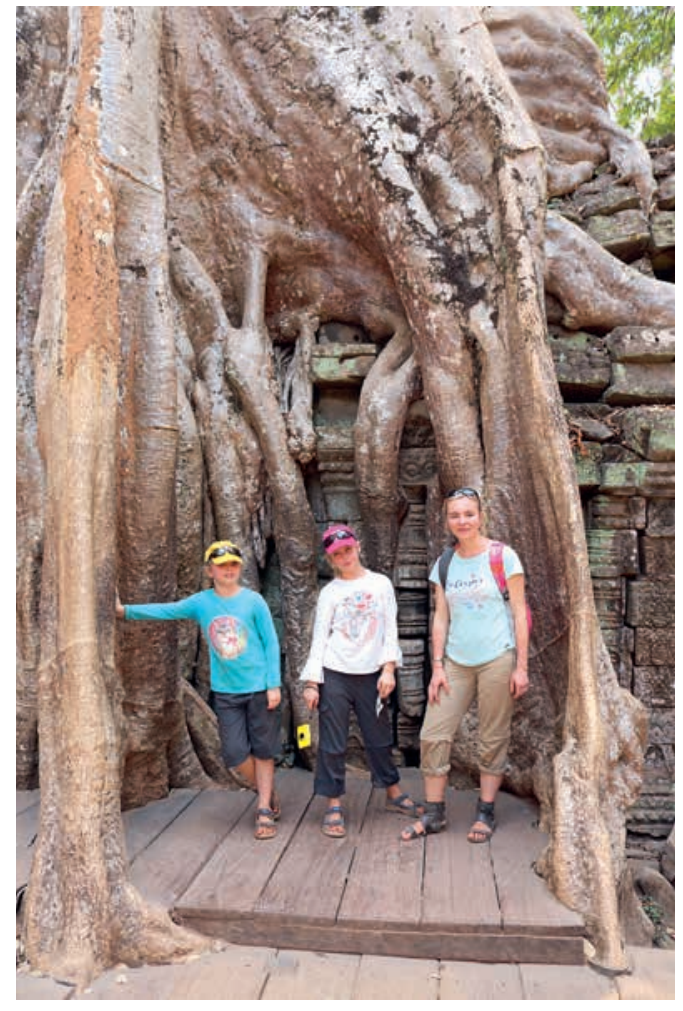

Abb. 15: Fototermin vor einem Spungbaum (Tetrameles nudiflora) in Ta Prohm. (Foto: H. Goetz) hinduistische Tempel aus dem 12. Jahrhundert wurde als einzige Anlage in dem Zustand belassen, wie ihn Archäologen vor mehr als 100 Jahren vorfanden - halb verfallen und von der Vegetation teils überwachsen. Um manche Gebäudeteile zu schützen und nicht zuletzt zur Sicherheit der Besucher wurden 2014 einige der Baumriesen gefällt. Doch nach wie vor zeigt sich die morbide Schönheit des Verfalls an allen Ecken und Enden. Viele teils eingestürzte Gebäude und Schreine sind von Dschungelpflanzen überwuchert, baumstammdicke Wurzeln krallen sich um Mauern und sorgen für ein faszinierendes Zusammenspiel von Kulturdenkmal und Natur. Durch das dichte Blätterdach der Baumkronen dringt mitunter nur diffuses Streulicht. Spektakulär nehmen sich etliche Exemplare von Tetrameles nudiflora aus (in der Khmer-Sprache heißen sie Spung), deren verzweigtes Wurzelsystem sich mitunter über zweistöckige Galerien spannt und diese fest im Griff zu haben scheint. Manche der archaisch anmutenden Baumgestalten haben meterhohe Brettwurzeln ausgebildet. Viel fotografiert wird ein von einem dichten Geflecht einer Würgefeige (Ficus virens) gerahmtes Eingangsportal, u. a. gab dieses die Kulisse für den Hollywoodfilm „Lara Croft:

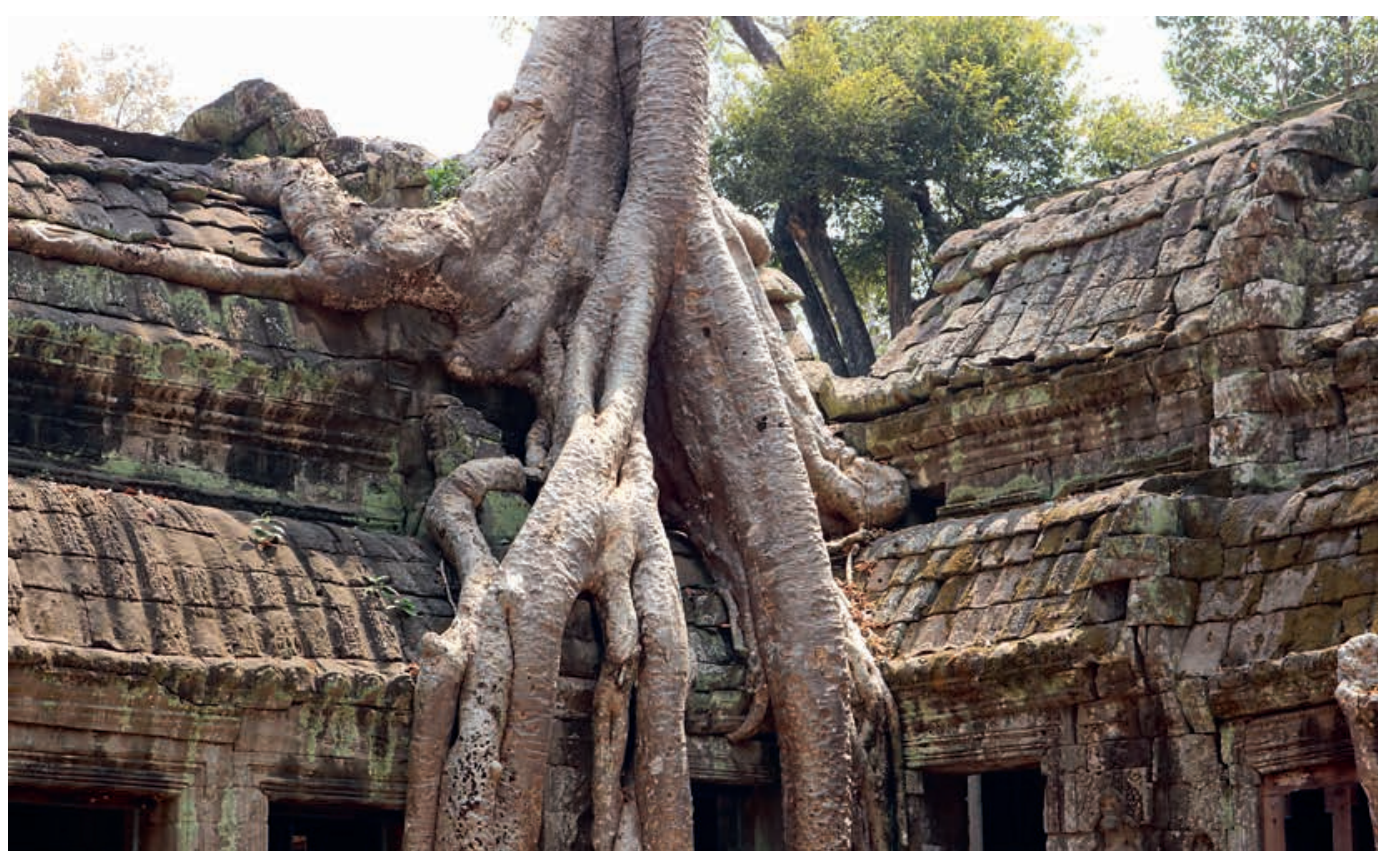

Abb. 16: Ein Spungbaum (Tetrameles nudiflora) hat die Tempelanlage von Ta Prohm fest im Griff. (Foto: R. Goetz) 
Tomb Raider“ (2001) mit Angelina Jolie in der Hauptrolle ab. In Anlehnung an den Hollywoodstreifen wird Ta Prohm in den sozialen Medien heute Tomb-Raider-Tempel genannt.

\section{Von Stink- bis Jackfrucht}

Nach dem Rundgang bzw. einer Rundfahrt durch das weitläufige Tempelareal landet man unweigerlich an einem der viele Straßenstände, an denen Kaltgetränke und lokale Snacks angeboten werden. Einheimische und Touristen gleichermaßen schätzen das erfrischende Wasser der Kokosnuss. Trinkkokosnüsse werden vor der Vollreife der Nuss geerntet, bevor sich das das Nährgewebe verfestigt hat; man trinkt das leicht süßliche Kokoswasser einfach mit einem Strohhalm aus der aufgeschlagenen Schale.

Das Angebot an tropischen Früchten ist saisonabhängig, viele der mundgerecht geschnittenen Früchte sind in Europa bislang kaum bekannt. So etwa die bis zu drei Kilogramm schwere Durianfrucht (Durio zibethinus). Deren Schale ist mit dicken Stacheln besetzt; innen ist die Sammelfrucht voll von kastaniengroßen Samen, die ein essbarer Samenmantel umschließt. Die Durianfrucht

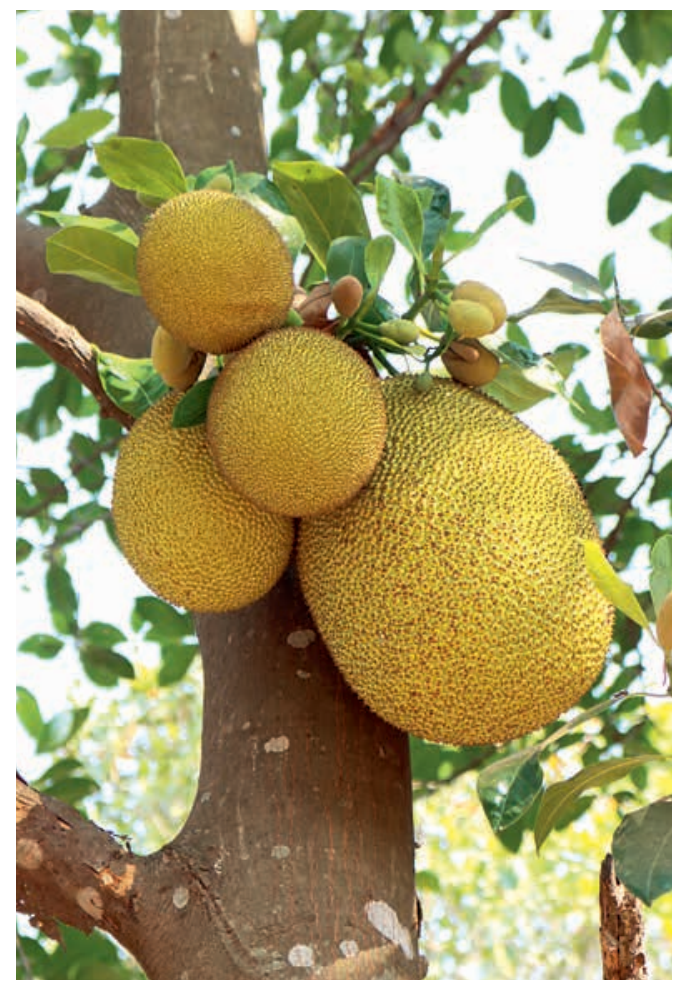

Abb. 18: Die Jackfrucht (Artocarpus heterophyllus) gehört weltweit zu den größten und schwersten Früchten. (Foto: R. Goetz)

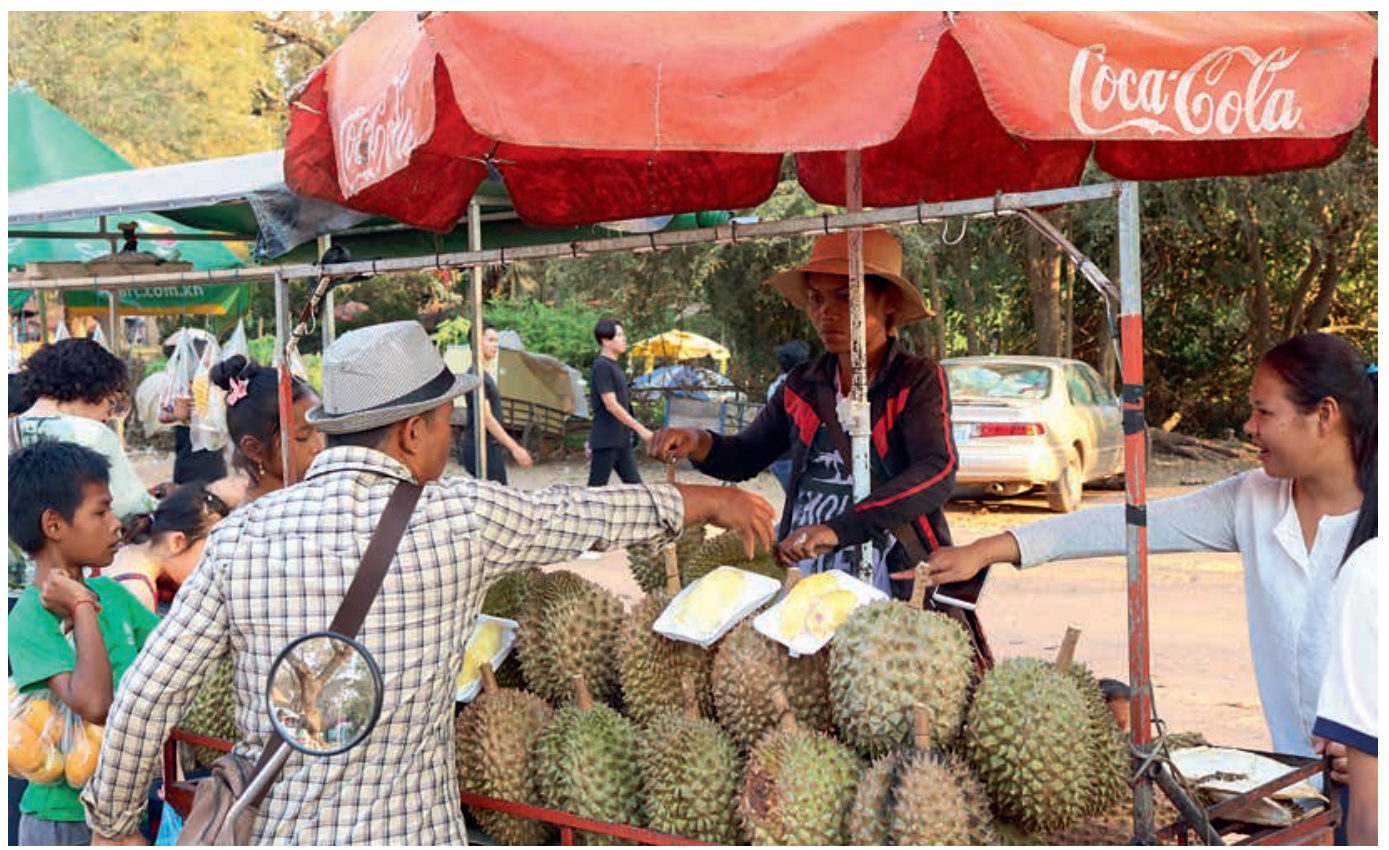

Abb. 17: Am Geschmack der Durianfrucht (Durio zibethinus) scheiden sich die Geister. (Foto: R. Goetz) 
wird nicht gerade schmeichelhaft Stinkfrucht genannt, in Südostasien gilt sie nichtsdestotrotz als der „König der Früchte“. Die meisten europäischen Touristen machen allerdings einen großen Bogen um sie - Geschmack und vor allem der durchdringende Kloakengeruch sind mehr als gewöhnungsbedürftig (Lieberei \& ReisdorfF 2007).

Ein ausgesprochenes Schwergewicht von mitunter mehr als 20 Kilogramm ist die Jackfrucht (Artocarpus heterophyllus), was auch der Grund dafür sein mag, dass von Konservenware abgesehen diese durchaus wohlschmeckende Frucht es bislang nicht auf den europäischen Markt geschafft hat. Gegessen wird davon nur der die Kerne umhüllende gelbfleischige Samenmantel. Je nach Saison finden sich an den Fruchtständen auch Rambutan (Nephelium lappaceum), Mangostane (Garcinia mangostana), Longan-Frucht (Dimocarpus longan) und Wasseräpfel (Syzygium

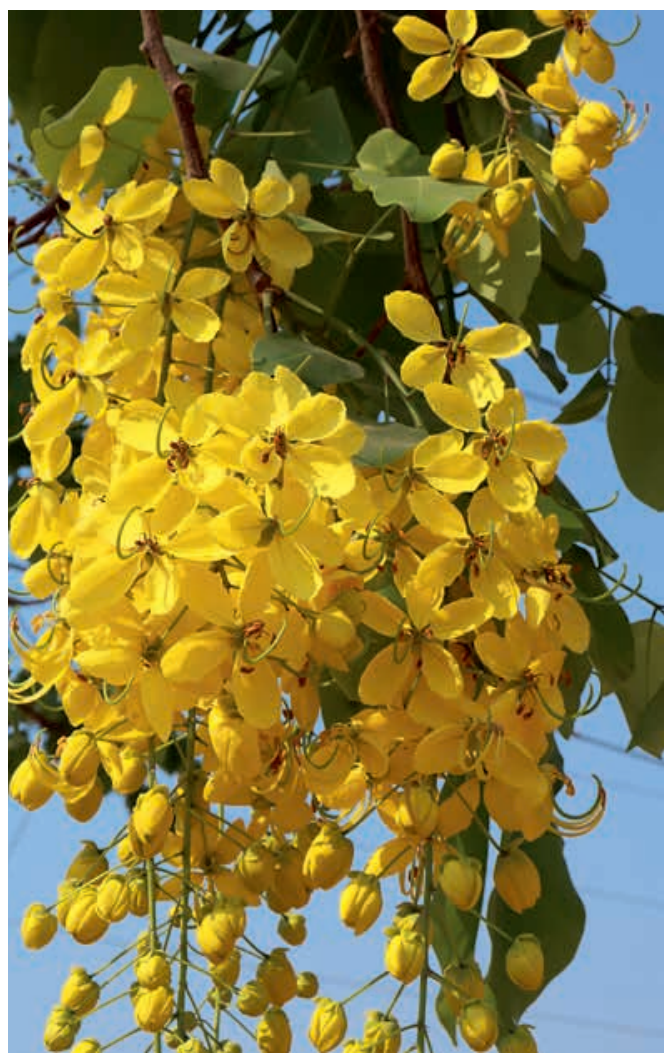

Abb. 19: Indischer Goldregen (Cassia fistula). (Foto: R. Goetz) mallaccense) sowie weitere bei uns kaum bekannte Arten.

\section{Frangipani, Goldregen \& Co.}

Der Frangipani aus der Gattung Plumeria ist sozusagen der Klassiker der hinduistisch-buddhistischen „Tempelflora“. In Angkor trifft man ihn jedoch relativ selten an, wohl aber im nur wenige Kilometer südlich gelegenen Siem Reap. Er ist dort in öffentlichen Parks zu sehen, u. a. den Royal Gardens vor dem Königspalast. Darüber hinaus zieren Frangipanibäume viele Privat- und Hotelgärten. In Indien und auf der Insel Bali steht das Hundsgiftgewächs (alle Pflanzenteile enthalten einen giftigen Milchsaft) vielerorts vor Tempeln, er wird von daher auch Tempelbaum genannt. Aus den intensiv duftenden weißgelben oder rosafarbenen Blüten werden Blütenkränze geflochten, die als „Offerings" (Opfergaben) bei Zeremonien und Festen Verwendung finden. Die Blütenstände befinden sich an den etwas plump aussehenden Astenden.

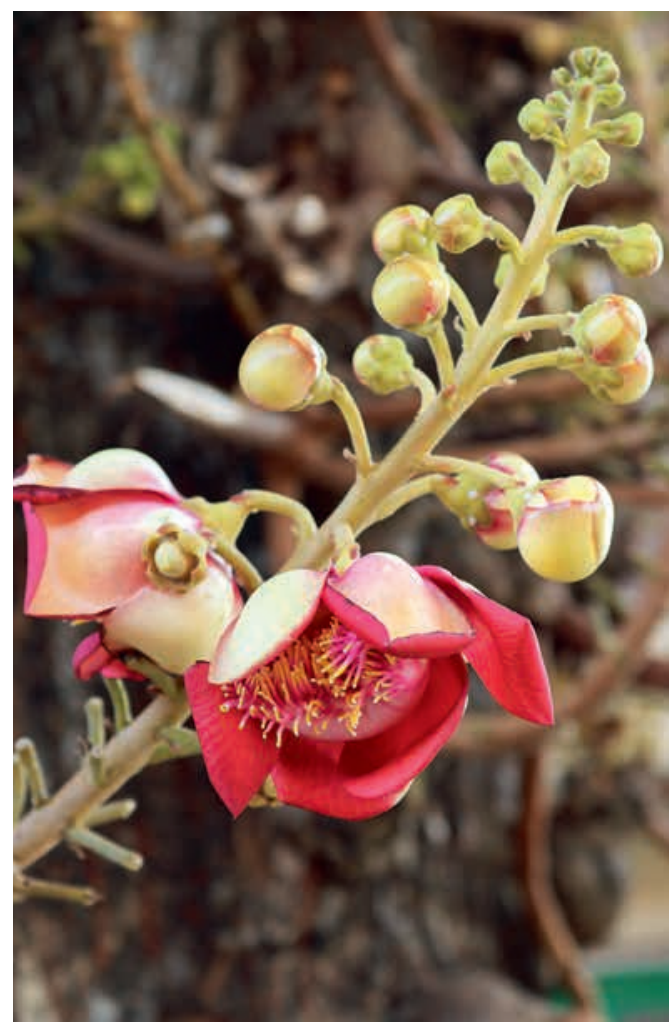

Abb. 20: Blütenstand des Kanonenkugelbaums (Couroupita guianensis). (Foto: R. Goetz) 
Ein charakteristisches Merkmal der Blütenblätter ist deren propellerförmig gedrehte Anordnung.

Während der Blüte von Februar bis Mai sticht der Indische Goldregen (Cassia fistula) mit in dichten Trauben von den Zweigen baumelnden goldgelben Blütenständen ins Auge. Die Fülle an Blüten macht ihn für Opfergaben geradezu prädestiniert Auffällig sind auch die wie dicke Schnüre von den Ästen herabhängenden Hülsen. Der Hülsenfrüchtler, er wird auch Röhrenkassie genannt, ist in Kambodscha ein viel kultivierter Straßenbaum, der bis zu $15 \mathrm{~m}$ hoch werden kann. Im Nachbarland Thailand hat die Kassie es zur Nationalblume gebracht.

Natürlich sieht man in Kambodscha genauso wie überall in den Tropen und Subtropen Kosmopoliten, etwa Bougainvillea (Bougainvillea glabra), Bauhinie (Bauhinia variegata) und Hibiskus ( $\mathrm{Hi}$ biscus rosa-sinensis). Ein Blickfang sind Vertreter aus der Familie der Rötegewächse (Rubiaceae), u. a. Ixora javanica, I. congesta und I. coccinea. Der immergrüne Strauch mit seinen orangeroten bis scharlachroten Blütenständen gehört in den Gärten Südostasiens zu den populärsten Zierpflanzen, von manchen Arten werden die Blüten zur Dekoration von Speisen verwendet.

Kurios mutet der aus den südamerikanischen Tropen vor etwa 200 Jahren nach Südostasien eingeführte Kanonenkugelbaum (Couroupita guianensis) an. Er fruchtet und blüht zur gleichen Zeit, sodass man neben den braunen „Kanonenkugeln“, den Früchten, auch die hübschen rosarot bis dunkelroten Blüten betrachten kann. Sie sitzen unmittelbar am Stamm, ihre Bestäubung erfolgt durch Fledermäuse (BärTEls 2013). Ein prächtiges Exemplar des Kanonenkugelbaums kann in der Hauptstadt Phnom Penh im Garten des Königspalasts bestaunt werden, der mit einem Baum der Reisenden (Ravenala madagascariensis) und nicht zuletzt seiner filigranen Pagodenarchitektur ein würdiger Abschluss ein jeder Kambodschareise ist.

\section{Literatur}

Ashton, P. 2014: In the forests of tropical Asia. - Kew.

Bärtels, A. 2013: Tropenpflanzen. - Stuttgart

BonN, G. 1997: Angkor - Toleranz in Stein. - Köln.

Chan, E. 2003: Handy pocket guide to tropical plants of Thailand. - Singapur.

Lieberei, R. \& Reisdorff, C. 2007: Nutzpflanzenkunde. Nutzbare Gewächse der gemäßigten Breiten, Subtropen und Tropen. - Stuttgart.

Loti, P. 1926: Eine Pilgerfahrt nach Angkor. - München.

Meyers, M. \& Markland, A. 2018: Kambodscha. - Ostfildern.

Mounot, H. 2000: Travels in Siam, Cambodia, Laos and Annam. - Bangkok.

RaY, N. 2018: Siem Reap \& the temples of Angkor. - London.

SAw, L.G. 2019: A naturalist's guide to the trees of Thailand and Southeast Asia. - Bangkok.

Schütt, P., Weisgerber, H., Schuck, H. \& Stimm, B. 2014: Bäume der Tropen. - Hamburg

WARREN, W. 2004: Handy pocket guide to the tropical flowers of Thailand \& Southeast Asia. - Singapur.

ZaHorka, H. 2018: Würgefeigen - Baumwürger und ihr geheimes Sexualleben. - Palmengarten 82: 25-30

\section{Internetseiten}

www.visit-angkor.org - die offizielle Webseite von Angkor www.gacp-angkor.de gibt einen Einblick in die Arbeit des deutschen Apsara-Konservierungsprojekts

\section{Anschrift des Autors}

Rolf Goetz, Rudolstädter Str. 121, 10713 Berlin, E-Mail: rolfgoetz@arcor.de 